\title{
Significado, desempeño y logro estudiantil en tareas instruccionales cotidianas
}

Paulo Volante

David Huepe

Carlos Cornejo

Pontificia Universidad Católica de Chile

\section{Resumen}

El objetivo de este estudio es investigar la relación entre el significado que los estudiantes elaboran respecto de sus tareas escolares y el nivel de desempeño que alcanzan en ellas. Basada en un enfoque de cognición situada, se diseñó una estrategia videográfica para elicitar, registrar e interpretar los significados que emergen cuando los estudiantes observan su propio desempeño a través de videos previamente filmados en su sala de clases. Las respuestas de los estudiantes fueron filmadas y sometidas a un análisis semántico y gestual. En los resultados se evidencian tres patrones de significado: automático, instrumental y proyectivo. Estos patrones sitúan a los estudiantes en tres cursos de acción diferentes, a pesar de que se trate de la misma tarea escolar. En particular, los estudiantes que desde la observación de sus tareas elaboran significados proyectivos, demuestran mejor comprensión, mejor desempeño y, a su vez, calificaciones superiores. En las conclusiones se destaca el valor metodológico y pedagógico del diseño de este estudio, pues facilita la comprensión de los diferentes significados que elaboran los estudiantes y evidencia cómo éstos influyen en el desempeño y logro educativo.

\section{Palabras clave}

Significado - Acción - Sentido - Comprensión - Gestos. 


\section{Meaning, performance and school success in daily instructional tasks}

Paulo Volante

David Huepe

Carlos Cornejo

Pontificia Universidad Católica de Chile

\begin{abstract}
The objective of this study is to investigate the relation between the meaning students attribute to their school tasks and the performance level they achieve in them. Based on the approach of situated cognition, a video strategy was defined to elicit, record, and interpret the meanings that emerge when students observe their own performance in scenes previously recorded in their classroom. The students' responses were taped and subjected to semantic and gestural analysis. Three patterns of meaning could be identified in the results: automatic, instrumental, and projective. These patterns place students along three different courses of action, despite dealing with the same school task. In particular, students that, from the observation of their tasks, create projective meanings demonstrate better understanding, greater performance and, eventually, higher qualifications. The conclusions highlight the methodological and pedagogical value of this project, since it helps to understand the different meanings created by the students, and reveals how these factors impact on school performance and success. (160 palavras)
\end{abstract}

\section{Keywords}

Meaning - Action - Sense - Understanding - Gestures. 


\section{Antecedentes teóricos}

En variados contextos sociales y cotidianos el comportamiento de los individuos parece homogéneo, regular y predecible. Por ejemplo, al observar estudiantes en sus tareas escolares en una sala de clases regulada y ordenada, éstos parecen "hacer lo mismo" cuando cumplen con las instrucciones de su profesor(a) o completan la actividad sugerida en su texto. Sin embargo, sabemos que la acción humana está mediada por el significado y que este emerge en la situación, lo que hace una diferencia sustancial entre la mera conducta observable y la acción orientada de los sujetos (Vygotsky, 2001; Bruner, 1998). Para capturar esta diferencia en la acción de los individuos, debemos ir más allá de la conducta y hacernos cargo simultáneamente tanto de la dimensión de sentido/significado como de la evolución y del contexto en que la acción se sitúa. Metodológicamente, ello implica acceder al discurso para comprender la historia que antecede y proyecta tales comportamientos.

Desde esta perspectiva, la acción se constituye en la unidad de análisis y la aproximación cultural en el método de estudio. Un ejemplo de esta aproximación es la planteada por Bruner (1998):

El supuesto fundamental de este tipo de psicología es, más bien, que la relación entre lo que se hace y lo que se dice es, en el proceder normal de la vida, interpretable. Esta psicología adopta la postura de que existe una congruencia públicamente interpretable entre decir, hacer y las circunstancias en que ocurre lo que se dice y lo que se hace. Es decir, existen relaciones canónicas establecidas por mutuo acuerdo entre el significado de lo que decimos y lo que hacemos en determinadas circunstancias, y esas relaciones gobiernan cómo conducimos nuestras vidas unos con otros. (p. 37)

De acuerdo a estos supuestos, es lícito y pertinente incorporar en la comprensión de la acción, al discurso sobre el comportamiento y al significado que emerge en la acción misma. En términos de F. Varela (1991) se trata de la oportunidad de estudiar la emergencia de procesos cognitivos tal cual como aparecen al sentido común, enfatizando la circularidad entre acción e interpretación:

Precisamente la mayor capacidad de la cognición viviente consiste en gran medida en plantear las cuestiones relevantes que van surgiendo en cada momento de nuestra vida. No son predefinidas sino enactuadas: se las hace emerger desde un trasfondo, y lo relevante es aquello que nuestro sentido común juzga como tal, siempre dentro de un contexto. (p. 89)

Lo relevante de este enfoque de la cognición, es que permite relacionar los procesos de construcción de significado, el desempeño de los sujetos y sus consecuencias en los diversos contextos de acción. Relaciones muy importantes a la hora de observar y evaluar diferencias en las capacidades manifiestas y en los cambios a nivel individual y grupal. Lo que a su vez refleja ciertos desarrollos actuales de la psicología cognitiva, que se ha planteado el desafío de realizar aproximaciones holísticas y ecológicas, que permitan comprender la acción orientada tal y como emerge en situaciones cotidianas (Cornejo; lbáñez; López, 2008; Freeman; Nuñez, 1999).

A partir de estas perspectivas teóricas, se destacan tres conceptos que guiarán la pregunta y la metodología de este estudio. El concepto de significado como eje de la relación entre discurso y comportamiento; el concepto de acción como distinción clave respecto al concepto de conducta, y la relevancia del sentido común a través del punto de vista de la primera persona.

\section{EI significado}

El cuestionamiento del supuesto de linealidad entre estímulos y respuestas como modelo de la acción humana, es un eje común 
de diversas perspectivas teóricas en ciencias sociales. Específicamente en psicología se ha planteado el problema del significado como una aproximación más comprensiva respecto a la cognición y al comportamiento motivado del ser humano (Bruner, 1998).

Desde este punto de vista el significado no es una propiedad inmutable de los estímulos, ni un contenido homogéneo dado por el ambiente, sino más bien, surge en la interacción entre el sujeto y el mundo, donde el nivel de explicitación de éste depende del grado de reflexión y automonitoreo que realiza el sujeto junto a otros (Valsiner; Van der Veer, 2000). Por lo tanto, no es que el esquema "estímulo - respuesta" no se cumpla en los seres humanos, sino que cuando buscamos acceder a la acción exclusivamente humana, y evolutivamente superior, este esquema no alcanza a dar cuenta de la complejidad de esta experiencia (Vygotsky, 2001).

Estos principios cognitivos que destacan el rol del significado, son especialmente relevantes en el contexto de situaciones de aprendizaje, ya que la condición activa del sujeto parece clave en los procesos de atención, selección y síntesis de las experiencias de aprendizaje. Esta idea confirma el supuesto que el significado que el sujeto elabora mientras realiza una tarea, tiene mucho que ver con la atención interna que despliega respecto lo que está haciendo y lo que quiere hacer. En este sentido, tales procesos cognitivos reflejan una actividad total del sujeto, en la que éste se involucra y se muestra, lo cual permite establecer la relación entre procesos internos y comportamientos observables:

\section{[...] prestar atención no es sólo analizar} con cuidado, sino que también implica un acto constructivo, que se expresa no sólo en las declaraciones o conductas específicas que podemos seccionar y puntualizar desde fuera, sino que puede ser interpretada desde la totalidad de su actuar en situación, esto es considerando su discurso verbal, gestos y posturas corporales. (McNeill, 1992, p. 19)

\section{Acción orientada}

De acuerdo a esta perspectiva, los límites de los procesos cognitivos se amplían más allá de lo que tradicionalmente hemos entendido como fenómenos de la mente. Aparece la dimensión corporal y sus movimientos como signos que despliegan y, al mismo tiempo, generan significado. De este modo la acción es situada, en tanto es orientada teleológicamente y en tanto está restringida y posibilitada por un contexto social. El contexto representa la dimensión cultural, pues se compone de elementos simbólicos y materiales que condicionan la acción y a su vez puede ser modificado por ésta, particularmente cuando surgen coordinaciones intersubjetivas:

En virtud de nuestra participación en la cultura, el significado se hace público y compartido. Nuestra forma de vida, adaptada culturalmente, depende de significados y conceptos compartidos, y depende también de formas de discurso compartidas que sirven para negociar las diferencias de significado e interpretación. (Varela, 1991, p. 29)

De este modo al considerar la acción como unidad de análisis, se intenta un ejercicio de comprensión holística que evita seccionar en conductas aisladas a los procesos cognitivos y considera a los resultados o consecuencias en el mundo como dimensiones de una misma experiencia. Por lo tanto para analizar la acción orientada se integra al discurso y a los actos, los cuáles se interpretan en una misma corriente de significado, en la que se revela la tensión entre la individualidad del agente y su pertenencia a un contexto de significados compartidos (Wertsch, 1998).

\section{La primera persona}

En consecuencia para comprender la acción orientada por el significado es necesario acceder al punto de vista de los sujetos que la realizan, lo cual metodológicamente supone una 
aproximación psicológica con un énfasis antropológico-cultural. En tal sentido, es preciso cuidar el acceso a la subjetividad, la validez ecológica de las observaciones, y la interacción en virtud de facilitar condiciones ideales de habla. Todo ello para hacer emerger lo más naturalmente posible a la primera persona, al agente, al sujeto, en tanto su discurso es la vía de acceso al componente intencional y consciente de la acción (Davidson, 2001).

De este modo, el lenguaje de la primera persona es una vía para acceder a los niveles de comprensión que, respecto de sí mismos, elaboran los sujetos en su acción situada y cotidiana. Tal como plantea Dreyfus (1996), siguiendo las nociones de Heidegger, es posible interpretar el grado de comprensión que alcanza el sujeto, en tanto no es considerada como proceso cognitivo interno e indescifrable, sino más bien, como una forma de vérselas en el mundo, una forma de dominio activo o simplemente un "saber hacer" en el contexto en que se esta situado.

Para ejemplificar esta noción de comprensión podemos seguir la analogía de Dreyfus (1996) respecto al dominio de una lengua extranjera, según la cual es posible diferenciar tres niveles de menor a mayor complejidad (Cuadro 1). En primer lugar el nivel más rudimentario, en segundo lugar el nivel acotado a lo conocido y tercer lugar su expresión más experta y creativa.

En este esquema se indica cómo el nivel de comprensión de los individuos puede relacionarse con diferentes niveles de acción en el mundo. Desde una operación circunscrita y acotada a un conjunto de reglas explícitas y elementos finitos (descifrar), a la posibilidad de manejar las condiciones de operación (interpretación) e incluso ampliarlas; hasta diversifi-

Cuadro 1
\begin{tabular}{|l|l|}
\hline Nivel de comprensión & \multicolumn{1}{|c|}{ Nivel de dominio } \\
\hline Descifrar & $\begin{array}{l}\text { Domina elementos del código, conoce } \\
\text { reglas formales. }\end{array}$ \\
\hline Interpretar & $\begin{array}{l}\text { Domina nociones de una lengua } \\
\text { extranjera, la usa deliberadamente } \\
\text { dependiendo del contexto y la tarea. }\end{array}$ \\
\hline Comprender & $\begin{array}{l}\text { Domina las destrezas de la lengua, } \\
\text { está habituado al ambiente y maneja } \\
\text { las irregularidades. }\end{array}$ \\
\hline
\end{tabular}

car las respuestas e incluso proponer respuestas creativas (comprensión).

Por otra parte, en la comprensión desplegada en la acción emerge el significado existencial, donde se expresa el ser ahí, en total coherencia con la narración que el mismo sujeto ofrece de sí mismo.

\section{Objetivo y pregunta del estudio}

Nuestro principal objetivo es 'comprender' los significados que emergen en la acción escolar, en el contexto de tareas cotidianas y desde el punto de vista de los estudiantes. Al mismo tiempo, se propone describir el significado que los escolares elaboran respecto su acción, para analizar las posibles relaciones con el nivel de logro en situaciones escolares. En este sentido, la pregunta que guía esta investigación, señala:

¿Cómo se relaciona el significado de la tarea con el desempeño del sujeto en el contexto de sus tareas escolares cotidianas?

En síntesis se intentará mostrar cómo la perspectiva del sujeto influye en la percepción de la tarea, la interpretación de ésta y en su desempeño. Para este objetivo el campo de la presente investigación lo constituyen escolares de enseñanza básica ( $8^{\circ}$ año enseñanza básica), que realizan la misma tarea instruccional en una jornada escolar regular sin ninguna intervención por parte de los investigadores.

\section{Diseño del estudio}

El objeto de análisis ha sido definido desde el paradigma del estudio de la acción, lo que supone una aproximación psicológica centrada en el significado y orientada culturalmente (Bruner, 1998), por lo tanto ha sido necesario idear un abordaje de tipo etnográfico que asegure la mayor validez ecológica y una interpretación de los datos lo menos fragmentada posible.

El campo de la investigación lo constituyen escolares que finalizan la enseñanza básica, cuya 
muestra fue definida por criterios de accesibilidad y relevancia (intencionada según los objetivos del estudio). El objeto de análisis es el significado elaborado por los escolares durante una entrevista estructurada, a propósito de su desempeño previo en una tarea instruccional realizada en la misma sala de clases.

Para facilitar el proceso de elaboración de significado, se diseñó un setting ad hoc y se ideó un proceso de registro para capturar información semántica y gestual simultáneamente. Este setting consistió en el uso de dos cámaras de vídeo, en dos momentos diferentes. Primero para la filmación en la sala de clases y luego, para una segunda filmación durante la entrevista individual, en la cual, los escolares se observaban a sí mismos a través de la filmación inicial y, simultáneamente, eran entrevistados con un protocolo de tres preguntas. Esquemáticamente el diseño se representa en la Figura 1 destacando las fases y episodios claves del proceso de elicitación de significado y registro videográfico.

En un primer momento (F1) nos introducimos en la sala de clases en una jornada regular, con previa autorización de los responsables de la escuela y de la clase. Sólo se seleccionó una sesión de matemáticas, por la mayor probabilidad de observar tareas instruccionales que requirieran ejercitación y cierto grado de desafío. Luego se consultó al profesor para identificar estudiantes de tres niveles de rendimiento en la asignatura: cuatro de alto nivel, cuatro de nivel medio y cuatro de bajo rendimiento. La muestra fue intencionada para incluir al menos 12 entrevistados que cubran el espectro de posibilidades de rendimiento esperado.

De este modo, previamente se instalaron cámaras para captar la dinámica del grupo completo, las instrucciones del docente y los ejemplos que éste desarrollaba en el pizarrón. Luego, durante la realización de la tarea en la sala de clases, se filmó focalizando a los estudiantes de la muestra. Para ello se protocolizó la filmación para cada sujeto, asegurando registrar su ubicación en la sala de clases, la postura corporal, el rostro, la mirada, la mano y su cuaderno durante el desempeño en la tarea.

Para el segundo momento (F2) se instaló un espacio para que cada estudiante observara individualmente su filmación, respondiera la entrevista y al mismo tiempo fuera nuevamente filmado. Este espacio fue diseñado para facilitar la recuperación de la experiencia anterior y se usó el vídeo filmado en la sala de clases, con el registro de cada

Figura 1: Setting del estudio

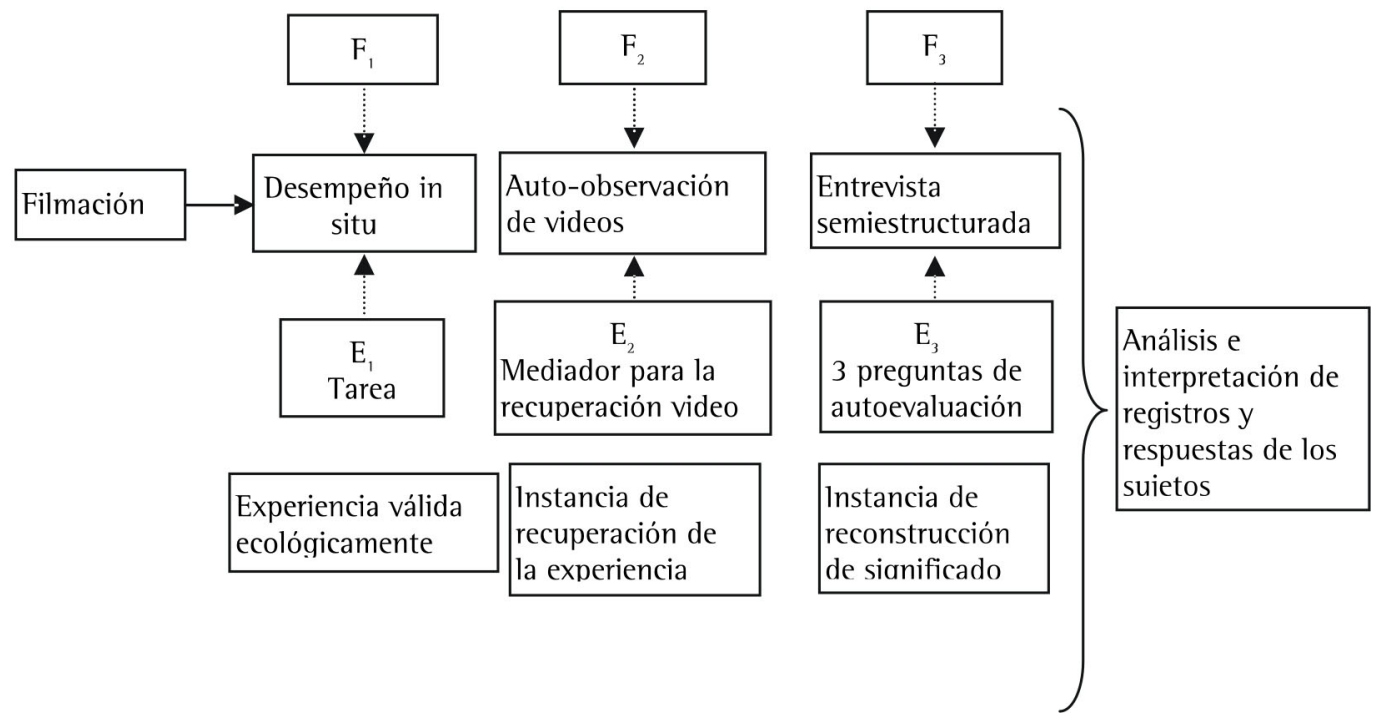


individuo (E2), como mediador para la recuperación y (re)elaboración del significado.

En este mismo espacio se realizó una entrevista estructurada de aproximadamente 12 minutos de duración cada una, la cual supone un tercer momento (F3) en paralelo a (F2). Durante esta situación los sujetos observaban su propio video, se les planteaba las mismas tres preguntas, y se reiteraban a lo menos tres veces para cada entrevistado. Las preguntas planteadas fueron las siguientes:

Pregunta 1: ¿Qué estás haciendo ahí?

Pregunta 2: ¿Para qué estás haciendo eso?

Pregunta 3: ¿Cómo sabes que lo estás haciendo bien?

En consecuencia, el espacio diseñado permite captar datos del desempeño grupal y de los individuos en su contexto natural (F1); luego facilita la recuperación de la experiencia (F2) y posteriormente permite la elaboración de significado en base a una breve entrevista protocolizada (F3). Posteriormente el análisis supone procesar datos videográficos y de contenido, integrando los registros verbales, corporales y del contexto.

\section{Estrategia de análisis}

El principal objetivo del plan de análisis, consiste en relacionar el significado elaborado por los estudiantes durante una secuencia de preguntas respecto su actividad en la sala de clases, su desempeño en la tarea y su rendimiento escolar.

De este modo, el protocolo de la entrevista fue pensado para facilitar la reflexión situada de los escolares y los videos filmados en la sala de clases para facilitar la recuperación de la experiencia y la elaboración de significado emergente, lo cual constituye la unidad de análisis del estudio. Por lo tanto, para definir la calidad del desempeño de los escolares, se utilizaron criterios externos e internos. Los primeros se basan en la evaluación del docente, quien identifica previamente a los estudiantes que considera de alto, medio y bajo rendimiento. Por otra parte, los criterios internos se refieren a la habilidad y fluidez que los escolares muestran durante la entrevista, tanto para explicar, como para ejemplificar la forma en que resuelven la tarea. Finalmente, el logro y rendimiento escolar también es un criterio externo al estudio, ya que se obtiene a través de los promedios de calificación que los estudiantes tienen durante el año escolar.

Adicionalmente al registrar las entrevistas en video, se pretendía contar con material para analizar la convergencia entre lo que el sujeto dice y lo que hace durante la entrevista, accediendo así a la versión en primera persona del presente, pasado y futuro de la acción de los escolares. En este sentido, se busca una interpretación de la acción situada, a partir del significado emergente, al que se accede a través de las palabras y los gestos de los entrevistados (McNeill, 1992).

\section{Presentación de resultados}

A continuación se presentan los datos obtenidos en las entrevistas, primero a nivel semántico se detalla el contenido de las respuestas a las tres preguntas y luego en base al registro videográfico se propone una descripción de claves gestuales. Finalmente se presenta una integración de ambos datos, para describir los significados de la acción inferidos en esta fase del estudio.

\section{Resultados a nivel semántico}

El primer conjunto de datos se obtiene a partir de la pregunta ¿Qué estás haciendo?, con la cual se pretende acceder a la percepción que los escolares pueden reportar de su actividad, ayudados por el registro de su imagen. De este modo, los estudiantes recuperaban la experiencia previa de clases y focalizaban su atención en dicha situación. Reiterando a lo menos tres veces la pregunta se obtuvieron inicialmente respuestas muy similares entre los estudiantes, resumidos en el siguiente texto: 
¿Qué estabas haciendo?

No sé... eso, la tarea..., lo que dijo la profesora, el ejercicio..., dividiendo..., las fracciones..., la prueba...

Las respuestas iniciales eran breves y reiteraban frecuentemente la expresión "no sé". Algunas describían el ejercicio dado por la profesora (fracciones) o simplemente usaban la denominación formal de la tarea ("ejercicio matemático", "prueba”). En la medida que la pregunta se repetía, algunos estudiantes dejaban las referencias formales a la actividad puntual que estaban observando y comenzaban a describir sus propios procedimientos para expresar el problema y su propia forma de resolverlo:

¿Qué estabas haciendo? (segundo intento) Sacando cuántas veces me cabe el 3 en $6 . .$.

/ Hay que poner dos ceros.../

Dividir para sacar el decimal /

Por ejemplo 4 dividido por 10.../ Transformar en decimal /

Como tienen tres ceros, le pongo así 1, 2, 3... / Ya... 10x10 tenía que sacar... 7 sacando primero las décimas

Tres de las cuatro estudiantes que reportaron procedimientos propios (heurísticos) presentaban mejores calificaciones en su desempeño escolar (matemáticas y lenguaje), además de ser calificadas como "alumnas de buen rendimiento" por sus profesoras.

A través de la siguiente pregunta, ¿Para qué estás haciendo eso? se intentaba acceder a la intencionalidad, al propósito, a la orientación y dirección que los estudiantes explicitaban al realizar la tarea. Las respuestas obtenidas en las primeras dos oportunidades que se hacía la pregunta también eran muy similares entre los escolares y seguían el siguiente patrón:

¿Para qué estás haciendo esto?

No sé / Porque hay que hacerla... / Para aprender... / Para hacer la tarea... / Para sacarme una buena nota... un siete/ ${ }^{1}$

Para saber más de matemáticas / Para no repetir de curso

Cabe indicar que del total de estudiantes entrevistados, cuatro de ellos en algún momento indicaron simplemente "no sé" frente a esta pregunta, de los cuales en tres casos su rendimiento en la asignatura era bajo y en un caso, rendimiento medio.

En la segunda reiteración de la pregunta ¿Para qué estás haciendo esto?, cinco estudiantes evocaron una respuesta de mayor profundidad, que iba más allá de la descripción de la tarea inmediata, expresando una orientación al futuro, en un horizonte amplio de posibilidades, como si emergiera una narración de tipo personal que trasciende la actividad puntual y cuyo contenido se resume a continuación:

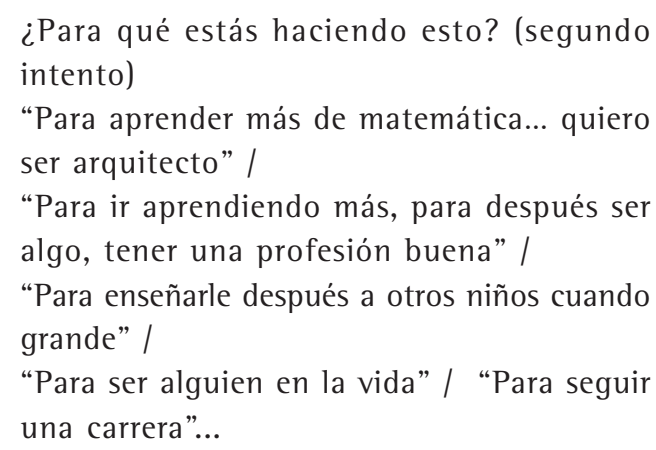

Cuatro de los cinco casos que reportaron estas respuestas correspondían a estudiantes de alto rendimiento y en su discurso fueron muy recurrentes las expresiones que referían a su proyecto personal y vocacional.

La última pregunta buscaba profundizar en la comprensión que los estudiantes podían explicitar respecto su actividad escolar, lo cual implica atender a los criterios que usaban para valorar su desempeño. De este modo, en las dos primeras secuencias de respuestas se observó una alta accesibilidad a la figura del profesor

1. El valor 7 se refiere a la máxima calificación que obtiene un estudiante en el sistema escolar chileno. 
como principal referente de la calidad de la tarea y del desempeño de los estudiantes:

¿Cómo sabes que lo estás haciendo bien? No sé... / No sé si está bien / Porque lo revisan en la pizarra /

Porque la tía me dice / Porque así lo enseñó la tía / Porque el profesor lo enseñó / me lo van a revisar/ Un compañero me enseñó /

La mayoría de los estudiantes se ajustaban a este patrón, que puede describirse como un continuo desde la percepción de no saber hasta la percepción de dominio basado en las instrucciones del docente. Varios estudiantes (5) inician su respuestas con "no sé", aludiendo a que no pueden dar cuenta por sí mismos del criterio o la regla para evaluar su desempeño. Luego surgen respuestas que refieren a un criterio externo y personalizado en el profesor, es decir, lo correcto o incorrecto está dado por lo que dijo o dirá el docente. Finalmente, en una nueva secuencia de preguntas y respuestas, emergen criterios propios, tales como la autopercepción de saber, la seguridad de haber ensayado previamente, la enunciación de una regla o simplemente la autoafirmación de sí mismo:

¿Cómo sabes que lo estás haciendo bien? Porque lo hemos hecho hartas veces / Porque estudié / Sé multiplicar y dividir / Por los ceros... / Porque sabía hacerlo

Lo común a las respuestas anteriores, es que se focalizan y se orientan en función de la tarea presente y de su ejecución en el contexto de la clase, de la ejercitación o de la claridad en el procedimiento empleado. Sin embargo, se observó otro patrón de respuestas en tres estudiantes que enfatizaban sentidos más allá de la tarea presente, explicitando un mayor horizonte temporal, rescatando una síntesis de experiencias pasadas y mostrando un mayor involucramiento y proyección personal desde la actividad actual:

¿Cómo sabes que lo estás haciendo bien? Lo había aprendido / Porque me estoy es- forzando en mis tareas / Yo le he puesto mucho empeño /

Porque estoy estudiando, me estoy esforzando.

Las tres estudiantes que manifiestan este patrón tienen coincidentemente un muy buen rendimiento escolar, siendo a su vez quienes resuelven la tarea con mayor fluidez y seguridad durante la entrevista. Este patrón de respuesta no se observó en estudiantes con bajo rendimiento, y parece ser un discurso altamente diferenciador dentro del grupo de entrevistados.

En síntesis, a partir del análisis semántico se observan patrones y diferencias relacionados con el significado explícito que los estudiantes elaboran respecto la tarea durante la entrevista. Se observa que existen, para la primera pregunta, tres tipos de respuestas, en primer lugar aquellas que "dicen" simplemente que no saben lo que hacen, o que aluden a estar haciendo "lo que hay que hacer", dado el contexto y dadas las instrucciones de la profesora. Estas respuestas se caracterizan típicamente por expresiones como: "no sé", "eso", "estoy copiando de la pizarra" o "[estoy haciendo] la tarea". En segundo momento, se registran respuestas que se acercan a una descripción de procedimientos matemáticos específicos: "dividir", "la fracción", "dividir para sacar el decimal", y también otras que aluden a la actividad escolar como "la prueba", "el ejercicio", "la guía". Por último, surge un tercer nivel en que se narra la forma personal de resolver la tarea, que incluso es más rica en términos "personales" que en descripciones operacionales. En estas respuestas, el sujeto parece describirse a sí mismo en el desempeño en la tarea: “...Veo cuántas veces me cabe el 3 en el 6 y entonces..."; ... “como tiene tres ceros la pongo asi".

La distinción de estos tres patrones de respuesta, se hace más evidente en la segunda pregunta (¿para qué lo haces?), pues en tal caso las respuestas del tipo "no sé" son más frecuentes, al igual que las respuestas que refieren a las instrucciones ("porque hay que hacerlo", "para la prueba”, "porque sí” o "para 
mostrárselo a la tía"). En un segundo tipo de respuestas, se alude más a los resultados esperados en la tarea, tales como: "para sacarme una buena nota”, "para sacar un 7 no más”, "para pasar de curso", "para no quedar repitiendo" o, simplemente, "para sacar el decimal". Finalmente, para el tercer patrón de respuestas se observa un discurso más amplio respecto de la situación inmediata, y que supone cierta proyección personal. En este tipo de respuestas se alude a las consecuencias esperadas en el futuro, en la medida que se logre el dominio anterior ("para sacar cuentas y todo", "para aprender matemática”, "para seguir una carrera”, "para ser arquitecto"..., "para ser alguien en la vida”). De este modo, este tercer nivel proyecta a los sujetos hacia múltiples posibilidades, a partir de la mayor o menor amplitud del campo de significado que elaboran en sus respuestas durante las entrevistas.

En este tercer nivel, la tarea y el desempeño en ella parecen ser un signo que indicaría posibilidades inciertas y que no son especificas, pero que parecen estar presentes en la acción que se realiza aquí y ahora. Esa acción es coherente con el contexto escolar pero se proyecta más allá de las condiciones y el desempeño actual. Entonces, cuando el entrevistador plantea la secuencia de preguntas, ¿Qué estás haciendo?, ¿Para qué lo haces?, y ¿Cómo sabes que lo haces bien?, sus respuestas no son una mera descripción de lo que se hace, sino que se completan con lo que se dice que hace, y este discurso parece enriquecido por un significado existencial que proyecta y guía su desempeño.

En palabras de los escolares, presentamos a continuación uno de los textos sobre los que descansa esta interpretación ${ }^{2}$ :

P: ... ¿Por ejemplo, ahí qué hay que hacer?

R: ya... 10×10 tenía que sacar, me daba 100 y el decimal como era dos..., tenía que ponerlo ahí... 0 y el 3 , y en fracción me quedaba así... tenía que ocupar el 3.

$\mathrm{P}$ : Entonces, ahí ¿qué estabas haciendo tú?
R: Yo... estaba sacando primero las décimas... multiplicando $10 \times 10$ para que me diera 100 , ese 100 para la fracción y para sacar el número decimal por cuanto se...

P: ¿Para qué haces eso... tú?

R: Yo... por... cómo, no entiendo por qué... ¡Ahí sí que no....!

P: ¿Para qué haces esa operación...?

R: A esto de aquí... $10 \times 10 \ldots$ porque el $10 \times 10$ me daba 100 para saber cuántos ceros le pongo al 10 ... y si está elevado a 2 y si está elevado a cuánto el 0 se multiplica...

$\mathrm{P}:$ ¿Y cómo sabes que eso está bien...?

R: Porque ....a ver... lo que había hecho... me lo habían explicado y entonces lo entendí tan bien... que me salió... así... primera vez... sí, porque a mi no me gustan las matemáticas $P: Y$ entonces, ¿para qué haces eso...?

$\mathrm{R}: \mathrm{mmm}$... para ir aprendiendo más, para después ser algo... tener una profesión buena... para no... para llegar a ser alguien... aunque no me gustan mucho las matemáticas...

P. ¿Y cómo sabes que lo haces bien, eso que tú quieres hacer?

R: Lo que yo quiero hacer?... porque, a ver .. Ud., me dice sobre el ejercicio o por lo que quiero

P: ¿Por lo que tú quieres hacer?

R: Porque estoy estudiando y quiero seguir más... después llegar a la universidad pa' tener una profesión

P: ¿Y cómo sabes que estás haciendo bien eso?

Porque... a ver... estoy estudiando, me esfuerzo harto pa' aprender... y ... no sé.....

En este breve texto es posible apreciar cómo evoluciona el significado de la tarea durante la entrevista y cómo progresivamente se diferencia a pesar de que las preguntas y la tarea instruccional a la que se refieren son exactamente iguales para todos los entrevistados. En este caso, se observa cómo el discur-

2. Donde P: pregunta del entrevistador y R: respuesta del entrevistad. 
so fluye desde la descripción de la operación hacia declaraciones que la trascienden y proyectan el sujeto hacia un futuro posible. Este patrón de respuestas, coincide en los casos de mayor rendimiento escolar y no se encuentra en los casos de menor rendimiento.

\section{Resultados a nivel de gestos}

Luego del análisis de contenido, se utilizaron los registros videográficos de las entrevistas como complemento al análisis semántico para obtener una aproximación más integral. Este material fue analizado observando gestos, posturas, ritmos, movimientos y señales pragmáticas de la comunicación, bajo la idea que "gestos junto con el discurso ayuda a constituir pensamientos" (McNeill, 1992, p. 245). Con esta orientación el análisis buscó detectar pautas de respuesta en las que los gestos agregaran evidencia para interpretar el discurso de los sujetos. De este modo, se encontró evidencia para proponer dos patrones gestuales predominantes. El primero asociado con respuestas seguras, regulares, relativas a información conocida y muy asociada al rol escolar predecible. Este patrón gestual fue interpretado como parte de un repertorio "funcional" y propio de un guión que da cuenta del rol de estudiante y del contexto tradicional de una sala de clases. En segundo lugar, se encontró otro patrón asociado a gestos irregulares y movimientos oscilantes, propios de respuestas inciertas, relativas a información desconocida ante la cual se requiere activar procesos de búsqueda y elaborar significado en tiempo presente. Este patrón sugiere un proceso microgenético (Rosenthal, 2005) que da cuenta del desarrollo de experiencias psicológicas que ocurren en el aquí y el ahora, durante un breve período de tiempo y que en este caso emerge en el desarrollo de la entrevista.

Esquemáticamente en la Figura 2 se representan imágenes tratadas de los entrevistados, para graficar las conceptualizaciones, y relaciones propuestas en este análisis. Se proponen dos ejes que describen un continuo de menos a más, y originan cuatro posibilidades que se identifican con cuatro casos representativos de las entrevistas realizadas.

Figura 2: Patrones gestuales

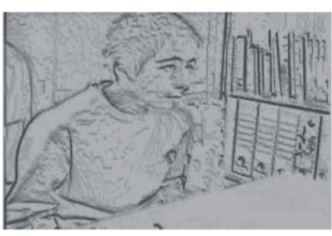

Gestos "No sé"

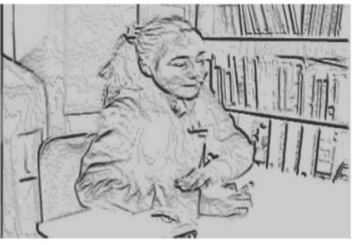

Gestos ejecutivos
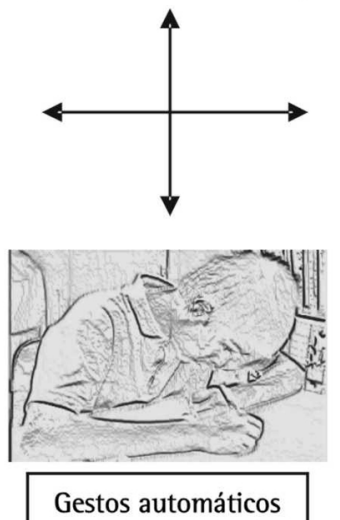

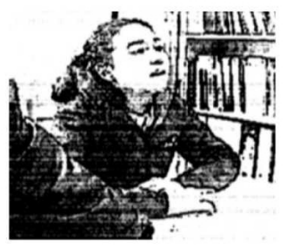

Gestos exploratorios 
En el eje vertical se representa la secuencia de gestos asociados a certidumbre y a seguridad en el desempeño y en el discurso. En el extremo inferior de ese eje se ubican gestos asociados a respuestas rápidas, con ausencia de quiebres, $\mathrm{y}$ movimientos focalizados en la tarea. En estos gestos se corresponden con discurso repetido, obvio, y prácticamente sobreentendido: gestos automáticos. Son movimientos que acompañan respuestas breves, sin mayor explicación y con dificultad para comunicar lo que se hace. En el mismo eje, pero en el extremo superior se repre-sentan gestos y movimientos de ejecución, focalizados en la tarea y con una alta coordinación entre rostro, mirada y mano: gestos ejecutivos. Son movimientos que acompañan respuestas rápidas, seguras y donde el entrevistado muestra dominio y control respecto a lo que hace y dice que hace.

En el eje horizontal, de la Figura 2, se representan los gestos en un continuo de mayor a menor ritmo y que interpretamos como grados crecientes de incertidumbre y exploración en la construcción de sus respuestas. En el extremo izquierdo los gestos indican pérdida del equilibrio, movimientos ondulatorios, miradas de rastreo de un lado a otro, literalmente como si se buscara una respuesta que no está en ninguna parte: gestos "no sé". En el extremo derecho, se observan gestos similares, movimientos de tronco y cabeza pronunciados, con quiebres y posturas de desequilibrio. Como si el cuerpo se acomodara, como si buscara una nueva posición hasta responder la pregunta. Nos parece que estos movimientos pueden interpretarse como procesos en que se está buscando y elaborando al mismo tiempo una respuesta: gestos exploratorios. Según este patrón, las posturas inestables, los movimientos horizontales y oscilantes, además de la primacía de miradas sin un foco claro, revelan un acceso incierto al significado de la acción y al mismo tiempo una elaboración de significado aquí y ahora. Por una parte, representan a aquellos que declaran que no saben lo que hacen, pero también se observan en los entrevistados que enfrentan las preguntas más allá de guiones conocidos y muestran gestos de un mayor involucramiento.
Por lo tanto, cuando los entrevistados enfrentan a las preguntas desde sus certezas se observan movimientos más regulares, generalmente en direcciones verticales: del ojo a la mano. El ritmo suele ser rápido y el discurso breve y sin quiebres. Esto hace pensar que tanto los procesos cognitivos automáticos como los ejecutivos, se reflejan en gestos lineales, repetitivos y que parecen estar basados en un guión conocido, propio del rol o de quien domina previamente la tarea. Por otra parte, los procesos reflexivos, en que se infiere elaboración y búsqueda de significado, se relacionarían con gestos asociados a la exploración, con movimientos más horizontales que verticales, con mayores quiebres en la posición corporal y miradas oscilantes.

\section{Análisis integrado de resultados}

Analizando el contenido de las respuestas y relacionándolo con su imbricación gestual, es posible establecer relaciones entre las respuestas de cada estudiante. De esto este modo, se observa una tendencia a la coherencia entre la respuesta textual, las señales corporales y el desempeño en las tareas observadas. De esta manera si interpretamos esta coherencia entre el decir y el hacer como el significado de la acción, y si a partir de este significado inferimos distintos tipos de comprensión e involucramiento en el desempeño escolar, entonces, podríamos dar cuenta de las diferencias de desempeño y logro desde los diferentes patrones de elaboración de significado capturados en las entrevistas.

Este análisis integrado, justificaría postular tres tipos de comprensión de la acción en los estudiantes. En primer lugar se propone un nivel de comprensión que puede ser descrito como “automático". Aquí prevalece el rol del escolar, las respuestas son acordes a las señales del contexto inmediato, ceñidas al presente y con dificultad para explicitarse verbalmente (casos: $1^{\circ}, 7^{\circ}$ y $4^{\circ}$ ). En segundo lugar, se observó un nivel que llamamos "instrumental", porque alude a significados 
que enfatizan la relación medios-fines, con respuestas que utilizan código explícitos de la tarea (matemática, fracciones, división) o la actividad contextualizada (el ejercicio, la guía, la prueba) y marcadamente orientadas al logro de un resultado deseable: sacarme un 7 , por las notas, para no quedar repitiendo (casos: $2^{\circ}, 8^{\circ}, 9^{\circ}$, $10^{a}$ ). Por último, un tercer nivel de comprensión, puede denominarse "proyectivo" porque manifiesta que la tarea actual está orientada en función de un futuro posible, comunicando un alto involucramiento y significados que van más allá del desempeño actual. Además, en los casos en que se expresa este nivel se muestra un mayor dominio en la tarea y un despliegue más rico del "saber hacer" de los estudiantes.

En el Cuadro 2 se agrupan los casos mencionados, se resumen sus respuestas y se describen gestos predominantes, así como y el nivel de desempeño en las tareas, además de su rendimiento en la asignaturas escolares.

De acuerdo a estos datos, los estudiantes que comunican un significado de tipo "proyectivo" $\left(3^{\circ}, 5^{\circ}, 6^{\circ}, 11^{\text {a }}\right)$ muestran un desempeño superior en la tarea y éste, a su vez, se relaciona con un mayor rendimiento escolar. Las posturas corporales de estos estudiantes también están relacionadas con este perfil, ya que muestran movimientos focalizados y de búsqueda simultáneamente, según respondan en función del dominio previo en la tarea o de la elaboración de significado durante su desempeño en ella.

\section{Conclusiones}

A partir del marco teórico y los datos recolectados, se proponen las siguientes conclusiones y se plantean preguntas para futuros estudios.

\section{a) En el mismo colegio, curso y clase: la} tarea no es la misma para todos

Aparentemente al observar las salas de clases en un día común y en una actividad cotidiana, todos los estudiantes parecen estar haciendo lo mismo: copian de la pizarra, atienden la instrucción y/o hacen la actividad, la guía, o la prueba que les entrega el profesor(a). Pero los comportamientos observables de los estudiantes, a los cuales accedemos por el registro de su ubicación, postura, ritmo y gestos - entre otras señales - nos muestran sólo una dimensión externa; indican la conducta social, que dado su carácter institucional tiende a conformarse y repetirse. Sin embargo, esa conducta no es suficiente para comprender la acción que realizan los estudiantes.

En este estudio, al preguntarles a los estudiantes ¿qué hacen? en un momento cualquiera de su actividad, se constató que sus respuestas eran inicialmente similares entre sí, pero luego se diferenciaban en la medida que se reiteraba y profundizaba la pregunta. En estos casos la mayoria de los entrevistados respondió: "estoy haciendo la tarea" o "estoy haciendo lo que la

\begin{tabular}{|c|c|c|c|c|c|c|}
\hline Casos & $\begin{array}{l}\text { ¿Qué estás } \\
\text { haciendo? }\end{array}$ & ¿Para qué? & $\begin{array}{l}\text { ¿Cómo sabes que } \\
\text { lo hiciste bien? }\end{array}$ & Gestos & $\begin{array}{l}\text { Desempeño } \\
\text { en tarea }\end{array}$ & $\begin{array}{l}\text { Rendimiento } \\
\text { promedio } \\
\text { (grupos) }\end{array}$ \\
\hline $\begin{array}{l}1^{\circ} \\
7^{\circ} \\
4^{\circ}\end{array}$ & La tarea ... & $\begin{array}{l}\text { Porque hay que } \\
\text { hacerla }\end{array}$ & $\begin{array}{l}\text { No sé si estoy bien/la } \\
\text { profesora las corrige }\end{array}$ & $\begin{array}{l}\text { Poco cambio de gestos } \\
\text { y posturas, predominio } \\
\text { de movimientos } \\
\text { lineales y verticales. }\end{array}$ & $\begin{array}{l}\text { Incorrecto } \\
\text { Incorrecto } \\
\text { Parcialmente } \\
\text { correcto }\end{array}$ & $\begin{array}{l}\text { Lenguaje: } 4,4 \\
\text { Matemática: } 4,3 \\
\text { Rendimiento } \\
\text { suficiente }\end{array}$ \\
\hline $\begin{array}{l}2^{\circ} \\
8^{\circ} \\
9^{\circ} \\
10^{\circ}\end{array}$ & $\begin{array}{l}\text { Dividir, la fracción, } \\
\text { la prueba }\end{array}$ & $\begin{array}{l}\text { Para la nota no } \\
\text { más/para sacarme } \\
\text { un } 7 / \text { para no } \\
\text { quedar repitiendo }\end{array}$ & $\begin{array}{l}\text { Porque lo hicimos } \\
\text { hartas veces/cuando } \\
\text { se corrige en la } \\
\text { pizarra. }\end{array}$ & $\begin{array}{l}\text { Movimientos } \\
\text { oscilantes, búsqueda } \\
\text { de acomodo, } \\
\text { movimientos } \\
\text { horizontales }\end{array}$ & $\begin{array}{l}\text { Incorrecto } \\
\text { Parcial Correcto } \\
\text { Parcial Correcto } \\
\text { Correcto }\end{array}$ & $\begin{array}{l}\text { Lenguaje: } 4,3 \\
\text { Matemática: } 4,2 \\
\text { Rendimiento } \\
\text { suficiente }\end{array}$ \\
\hline $\begin{array}{l}3^{\circ} \\
5^{\circ} \\
6^{\circ} \\
11^{\circ}\end{array}$ & $\begin{array}{l}\text { Cuántas veces me } \\
\text { cabe el } 3 \text { en } 6 . . . \\
\text { sacando primero } \\
\text { las décimas ... }\end{array}$ & $\begin{array}{l}\text { Para ser alguien en } \\
\text { la vida/para sacar } \\
\text { una carrera/para } \\
\text { ser algo }\end{array}$ & $\begin{array}{l}\text { Porque sé/porque } \\
\text { estudié/porque me } \\
\text { estoy esforzando }\end{array}$ & $\begin{array}{l}\text { Gestos y posturas } \\
\text { lineales combinado } \\
\text { con movimientos } \\
\text { oscilantes. }\end{array}$ & $\begin{array}{l}\text { Correcto } \\
\text { Correcto } \\
\text { Correcto } \\
\text { Correcto }\end{array}$ & $\begin{array}{l}\text { Lenguaje: } 6,5 \\
\text { Matemática: } 6,0 \\
\text { Rendimiento muy } \\
\text { bueno. }\end{array}$ \\
\hline
\end{tabular}


profesora dijo", es decir, ejecutan el desempeño esperado de un escolar en el contexto instruccional. Una vez reiterada la pregunta, las respuestas describían procedimientos: "trato de resolver un ejercicio" o "tengo que transformar el número en potencia a decimal y fracción”, es decir, se da cuenta del propio proceso y del resultado esperado en la tarea. En algunos casos este nivel se describe con mayor precisión, porque se da cuenta de las reglas formales de la operación, e incluso incluye el planteamiento de una fórmula personal, una suerte de heurística que revela la comprensión instrumental de la tarea. Este tipo de respuestas está en la intersección entre este segundo nivel y el tercer tipo de comprensión donde los sujetos elaboran un mayor significado personal, y proyectan su hacer más allá del desempeño actual. En este tercer nivel, las respuestas a la pregunta ¿Qué haces aquí?, suponen un involucramiento y una proyección personal, que revela la acción orientada por inciertas posibilidades de futuro.

\section{b) El significado y el desempeño}

Una forma de explicar las diferencias en la percepción de la tarea, se revela en los distintos niveles de comprensión que los entrevistados muestran al responder la pregunta: ¿para qué haces eso? Los significados que emergen en estas respuestas explicarían la diferencia en los propósitos, en la dirección del hacer; es decir, revelaría al agente que experimenta diferencialmente las mismas tareas y a su vez guía el desempeño en el contexto escolar.

En un primer nivel las respuestas al para qué, son del tipo "porque estoy en clase de matemática”, "porque es lo que hay que hacer" y/ o "porque la profesora dijo". Indicando que el desempeño está guiado por la instrucción del docente y se limita al cumplimiento de un guión conocido, automático e innecesario de explicitar. En un segundo nivel, las respuestas se expresan en términos de una relación de medios y resultados, tales como: "para sacarme un siete", "para resolver el ejercicio", "para sacar el resultado”, "para transformar las números...” y/o "para aprender más”. De estas declaraciones, se deduce un predominio de un nivel instrumental que refiere a producir un resultado, realizar ciertas operaciones y aplicar procedimientos conocidos para obtener el logro esperado.

$\mathrm{Al}$ insistir en la pregunta, para alcanzar un nuevo nivel de reflexión, las respuestas al "para qué haces eso" se tornan más disímiles. Varios escolares optan por un "no sé", otros repiten fórmulas anteriores, manteniendo su análisis en el nivel instrumental. Sin embargo, algunos estudiantes parecen ver en su acción posibilidades futuras, fines de largo plazo y absolutamente impredecibles: "para sacar una carrera", "para ser algo", "para ser alguien en la vida". En este nivel se alude a propósitos que progresivamente amplían las posibilidades del significado y conectan el actuar cotidiano con una perspectiva futura, que parece anclada en un pasado de esfuerzo y un presente de alto involucramiento personal. En este sentido, cuando los estudiantes responden desde este tipo de comprensión respecto a lo que hacen en la sala de clases, nos parece que su acción "trasciende" la tarea inmediata y se proyecta más allá del deber escolar cotidiano.

\section{c) Modelos de aprendizaje: de la conducta a la} acción

En la tercera pregunta del protocolo se consultaba a los escolares ¿cómo sabes que lo haces bien?, aludiendo a la posibilidad del entrevistado para autoevaluar el desempeño y explicitar el significado de este. Una de las interpretaciones más sugerentes a estas respuestas permite inferir el modelo de aprendizaje que subyace en los estudiantes cuando dan cuenta del grado de control percibido de su desempeño. De este modo, varias respuestas parecen un ejemplo de imitación irreflexiva, dado que la actividad simplemente se justifica en función de una señal externa, del tipo: "no sé si estoy bien, pero la profesora dijo...", o "porque la profesora lo revisará", o más gráficamente "si le ponen un? (signo de aprobación)” o "porque estaba en la pizarra”. En este tipo de respuestas la posibilidad 
de comprobación es siempre externa, depende del profesor (a)o de algún código que se asume como una "señal dada". En este nivel el control del desempeño es totalmente heterónomo, lo que refuerza la idea de una comprensión automática, basada en el rol escolar y situada en un contexto que provee signos cuyo significado está dado y acotado institucionalmente.

En un segundo nivel de respuestas, los estudiantes aluden a su experiencia previa, al refuerzo positivo del profesor y en general a las consecuencias o recompensa esperada. Los estudiantes que elaboran estas respuestas, parecen pensar como un sujeto del "condicio-namiento operante”, cuyos significados están dados por las calificaciones del profesor y por la evitación del refuerzo negativo: "porque si me saco un siete está bien", "porque si no me saco un rojo", "porque si le pongo empeño me va bien". Es interesante destacar que aquí los estudiantes comunican la importancia que tiene el feedback del profesor y el valor que asignan a la calificación obtenida en sus tareas. Las calificaciones en estos casos, representan la única señal confiable de si lo está haciendo bien o no, lo que sugiere la necesidad de resignificar el uso docente de la calificación escolar, por lo menos en algunos hitos del proceso escolar. Ya que los estudiantes, que expresan este patrón de significado, parecen utilizarlas como una guía efectiva de su desempeño.

Un tercer nivel de comprensión se aproxima a experiencias de aprendizaje más autónomas. Aquí los escolares revelan su comprensión de los criterios operativos de los ejercicios y de sus reglas, pero también responden en función de su know how como estudiantes y en base a la confianza en su esfuerzo: "porque sé dividir y multiplicar"; "sé que está bien", "porque estoy estudiando y me estoy esforzando". Nuevamente, a pesar que en estos casos prima un énfasis más autónomo, no por ello se prescinde de la retroalimentación del profesor(a), el cual también está presente en las primeras respuestas de estos mismos sujetos.

Particularmente interesante son los casos en que los estudiantes no expresan ningún criterio o forma de automonitoreo de su desem- peño, limitándose a decir: "no sé si está bien", "no sé", "no sé... lo van a revisar..." respuestas muy usuales en quienes no resuelven correctamente los ejercicios y a quienes les es muy difícil expresar el "para qué" de su actividad. En estos casos las declaraciones parecen reflejar procedimientos automáticos y un comportamiento basado en recursos implícitos o no accesibles a la comprensión de los sujetos. En el otro extremo, se observan respuestas donde el saber si se está o no en lo correcto refiere al esfuerzo invertido y a los fines que parecen orientar el desempeño total del sujeto: "porque he estudiado harto", "porque me esfuerzo bastante" y "porque sé que si le pongo empeño me va a ir bien". En estos casos "el lugar" en que se verifica el conocimiento se describe desde la propia conciencia del desempeño y del compromiso personal, revelando con nitidez el patrón de significado proyectivo.

\section{d) Una vez, otra vez y otra vez más: el} significado emerge en la acción

La evidencia sobre los tres niveles de comprensión de la actividad escolar muestra que existe un incremento paulatino en la infor-mación personal en las respuestas en la medida que se reiteran las preguntas. Al preguntar por segunda o tercera vez ¿qué estás haciendo ahí? parecía cambiar el campo, accediéndose progresivamente a un nivel más personal, cómo si al ver su lugar en la sala, sus posturas y expresiones, se les facilitara la comprensión de su acción. Por lo tanto, nos parece que las condiciones de elaboración de significado que propone la entrevista se logran en la medida que se brinda a los estudiantes la posibilidad de reflexionar repetidamente sobre su desempeño, pues la reiteración de la pregunta conecta distintos niveles de significado. Cada secuencia parece facilitar la autocomprensión, provocando que se vuelva a mirar y se involucre con posibilidades más allá del desempeño actual. El proceso se ve facilitado aún más por el hecho que la metodología exigía que los alumnos observasen su propio desempeño en un video 
previamente filmado en condiciones espontáneas dentro del aula.

En este sentido, se constata la pertinencia de un concepto enactivo de significado (Varela, 2000), ya que los registros de las entrevistas muestran empíricamente la fluidez, diferenciación y progresiva complejidad que emerge en la medida que los escolares construyen sus respuestas, a pesar de estar expuestos al mismo protocolo y contexto de interacción.

\section{e) ¿Podemos observar cómo el significado media el desempeño?}

En la Figura 3 se sintetizan las relaciones claves que se espera encontrar entre significado, desempeño y logro, las cuales sugieren un posible modelo a probar empíricamente en futuras investigaciones. Las relaciones propues-tas en este último esquema muestran que ante una misma tarea instruccional y en condiciones similares, la comprensión y el desempeño en la actividad se diferencian considerablemente. Estas diferencias se interpretan como distintos patrones de significado que guían la acción de los entrevistados.

En el caso de los patrones de respuesta automática es muy probable que la comprensión de las tareas se limite a repetir instrucciones 0 simplemente a ejecutar la tarea y por tanto el desempeño y el resultado estarán muy guiados por la instrucción externa. Por otra parte, en el patrón de respuesta instrumental el desempeño esperado será superior y más ágil, puesto que el estudiante esta guiado por su orientación al resultado aún cuando la tarea no sea especialmente relevante o satis-factoria. Finalmente, el patrón que hemos llamado proyectivo refleja la orientación a la realización de fines que trascienden las tareas y sus instrucciones.

Es probable que este último patrón sea el que más representa la idealización del mejor estudiante. Sin embargo, los resultados muestran que los patrones de significado menos idealizados, tales como el automático o instrumental, también están presentes en los escolares de mayor desempeño y mayor logro. Lo que muestra que los patrones identificados no son estáticos sino factibles de evolucionar, y por tanto la comprensión de su dinámica es un desafío para investigaciones en desarrollo e intervención psicoeducativa.

Finalmente, a la hora de proponer intervenciones situadas en el contexto escolar, nos parece necesario comprender y utilizar el punto de vista del estudiante, pues a pesar de los esfuerzos por proveer oportunidades equivalentes para todos los escolares, estos siempre elaboraran significados diferenciados que a su vez influirán en el desempeño y logro de las organizaciones educativas.

Figura 3: Relaciones entre sentido, desempeño y logro.

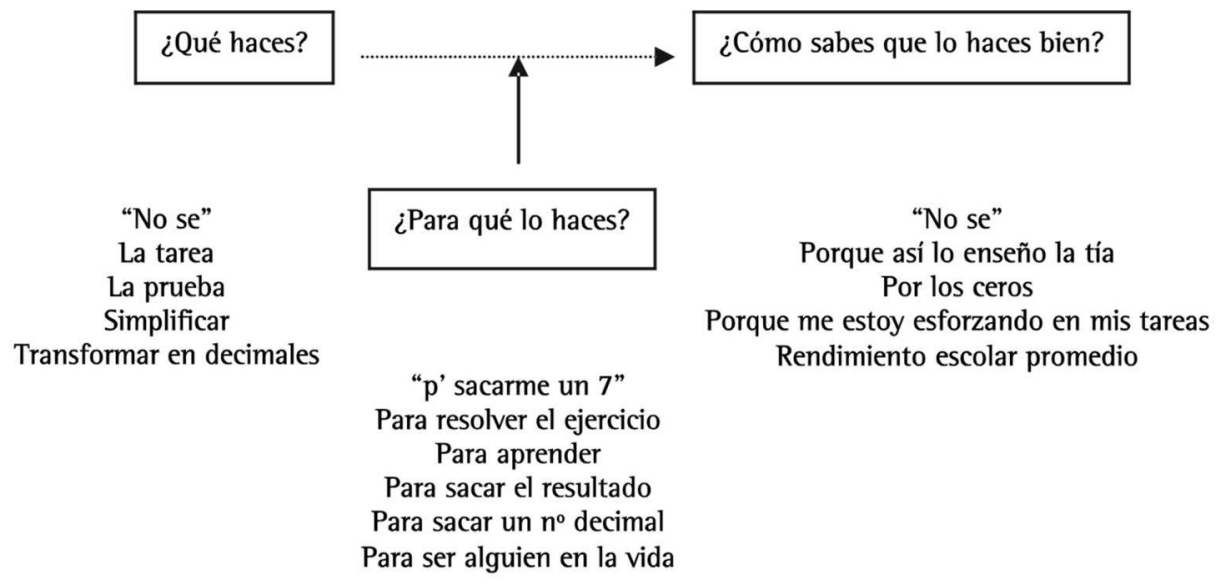




\section{Referencias bibliográficas}

BRUNER, J. Actos de significado. Madrid: Alianza, 1998.

CORNEJO, C., IBÁÑEZ, I., LÓPEZ, V. Significado, contexto y experiencia: evidencias conductuales y electrofisiológicas del holismo del significado. En: KRONMÜLLER, E.; CORNEJO, C. (Eds.). Ciencias de la mente: aproximaciones desde Latinoamérica. Santiago: JCSáez Editor, 2008. p. 209-240.

DAVIDSON, D. The myth of the subjective. En: DAVIDSON, D. (Ed.). Subjective, intersubjective, objective. Oxford: Clarendon Press, 2001.

DREYFUS, H. L. Ser-en-el-mundo. Santiago, Chile: Editorial Cuatro Vientos. (1991 MIT Press), 1996.

FREEMAN, W.; NUÑEZ, R. Restoring to cognition the forgotten primacy of action, intention and emotion. In: NÚÑ̃EZ, R.; FREEMAN, W. (Eds.). Reclaiming cognition: the primacy of action, intention and emotion. UK: Imprint Academic, 1999.

McNEILL, D. Hand and mind. London: The University of Chicago Press, 1992.

ROSENTHAL, V. Microgenesis, immediate experience and visual processes in reading. In: CARSETTI, A. (Ed.). Seeing, thinking and knowing. Dordrecht: Kluwer Academic Publishers, 2004. p. 221-243.

VARELA, F. J. Conocer: Cartografía de las ciencias cognitivas. Barcelona: Gedisa, 1991.

El fenómeno de la vida. Santiago: Dolmen, 2000.

VALSINER, J.; VAN Der VEER, R. The social mind: construction of the idea. USA: Cambridge Universtity Press, 2000.

VYGOTSKY, L. S. Pensamiento y lenguaje. Obras Escogidas, v. 2. Madrid: Visor, 2001. (1935)

WERTSCH, J. V. La mente en acción. Buenos Aires: AIQUE, 1998.

Recibido en 04.03 .08

Aprobado en 07.04.09

Paulo Volante es $\mathrm{PhD}(\mathrm{c})$ en Psicología, Programa de Doctorado Escuela de Psicología y Profesor de la Facultad de Educación de la Pontificia Universidad Católica de Chile.

David Huepe es PhD(c) en Psicología, Programa de Doctorado Escuela de Psicología de la Pontificia Universidad Católica de Chile.

Carlos Cornejo es PhD Profesor Asociado del Programa de Doctorado de la Escuela de Psicología de la Pontificia Universidad Católica de Chile. 